\title{
Avaliação de mudança em processos terapêuticos
}

\author{
Elisa Mediei Pizão Yoshida \\ Pontifícia Universidade Católica de Campinas
}

\begin{abstract}
Resumo
O estudo das psicoterapias tem sido marcado pelo uso de métodos de investigação intensiva do processo terapêutico voltados para a compreensão dos fatores que facilitam a mudança. Baseiam-se, usualmente, na análise intensiva de caso único, identificando regularidades características dos estados mentais, padrões de conduta, assim como interacionais, que são associadas com mudança. Contam com instrumentos de avaliação desenvolvidos segundo referencial teórico específico em que conceitos metapsicológicos são definidos operacionalmente. Dependem, em geral, da avaliação de juizes independentes, experientes na técnica avaliada, que se baseiam em vídeos e / ou áudios das sessões de terapia, além de considerarem o contexto. Têm permitido o estudo exaustivo de processos terapêuticos e a obtenção de julgamentos baseados em construtos que evidenciam validade preditiva, além de gozarem de precisão entre avaliadores. A título de ilustração apresentam-se pesquisas envolvendo as seguintes escalas: "Defense Mechanisms Rating Scales", "Rutgers Psychotherapy Progress Scale" e "Escala Diagnóstica Adaptativa Operacionalizada - Redefinida".

Palavras-chave: mudança em psicoterapia / delineamento de pesquisa de caso único / pesquisa do processo terapêutico / pesquisa em psicologia clínica.
\end{abstract}

\section{Assessment of change In therapeutic processes}

\section{Summary}

The study of psychotherapies has been marked by the use of methods of intensive investigation of the therapeutic process, turned to the understanding of the factors that favor the change. Those methods are usually based on the intensive analysis of a single case, identifying regularities characteristic of mental states, behavioral and interacting patterns which are associated with change. They count upon assessment tools developed according to specific theoretical standards in which metapsycological concepts are operationally defined. In general, they depend on independent judges familiar with the assessed technique, who base their assessment upon videos and/or audio equipment of the therapy session, besides considering the context. They have allowed the exhaustive study of therapeutic processes and the obtaining of judgment based on constructs which evince predictive validity, besides getting reliability among raters. By way of illustration some researches are presented involving the following scales: "Defense Mechanisms Rating Scales", "Rutgers Psychotherapy Progress Scale" and "Escala Diagnóstica Adaptativa Operacionalizada- Redefinida".

Key words: change in psychotherapy / single case research design / research of the psychotherapeutic process / research on clinical psychology.

As pesquisas de processo terapêutico já contam com uma longa tradição no âmbito internacional, em que desde a década de 50, Rogers e seus associados buscavam compreender "o que é que acontece realmente na terapia com êxito? Quais são os processos psicológicos por meio dos quais se dá a modificação?" (Rogers, 1951/1974). Na verdade, estas e outras questões

Endereço para correspondência: Av. Francisco de Assis Dinis. 220, Parque dos Príncipes. Osasco-SP. CEP 06030-380, E-mail: eyoshida@telnet.com.br tel.: (011) 99487727 (res.), (019) 230-7180 (PU C-Campinas) 
continuam atuais apesar de todo o esforço realizado no campo da pesquisa em processos psicoterápicos.

Quando se observa a produção de pesquisa em psicoterapias na atualidade, verifica-se que a compreensão dos aspectos que contribuem para a mudança continua a ser um desafio. O objetivo continua a ser o de identificar os fatores específicos envolvidos no processo terapêutico e que sejam capazes de facilitar ou ensejar a mudança (Goldfried \& Wolfe, 1996).

Já se conta, por exemplo, com um acúmulo de evidências fornecidas por pesquisas de resultados que indicam que as psicoterapias são:

1. Efetivas;

2. que elas são mais efetivas do que ausência de terapia ou intervenções placebo;

3. que muitos pacientes que melhoram na terapia mantêm a melhora por um longo período de tempo;

4. que as psicoterapias, independentemente das orientações teóricas que as inspiram, parecem ser capazes de produzir mudança (Lambert, 1991).

Alguns fatores têm sido sistematicamente indicados como responsáveis pelos resultados favoráveis observados em processos terapêuticos conduzidos segundo técnicas muito diferentes entre si e com pacientes apresentando, igualmente, condições muito diversas. É consensual, por exemplo, o reconhecimento do valor da aliança terapêutica positiva sobre a qualidade dos resultados obtidos. Um segundo fator refere-se à capacidade demonstrada pelo paciente para lidar com situações difíceis no passado e que pode ser traduzido como indicação de sua capacidade de adaptação. Um terceiro fator reconhecido como facilitador de sucesso em psicoterapia, seria a capacidade do paciente de reconhecer o caráter psicológico de suas dificuldades, ou ainda, um quarto, sua motivação para a mudança (Messer \& Warren, 1995).

Observados em conjunto, estes fatores parecem indicar que os resultados da terapia dependem, em muito, dos recursos do paciente. Mas não se deve esquecer, que estes se atualizam sempre dentro do contexto da díade representada pelo paciente em relação com o terapeuta. Nesta medida, pensar em mudança em psicoterapia, significa considerar a dinâmica que se estabelece entre estes dois protagonistas do processo. Dentro deste quadro, portanto, procurar compreender em que circunstâncias a relação terapêutica leva à mudança e como ela ocorre nos diferentes tratamentos, surge quase que como uma decorrência natural de um processo de investigação (Greenberg, 1994).

Os trabalhos visando avaliar a eficácia das terapias, a partir de medidas obtidas no início e no fim dos atendimentos, vêm dando lugar a estudos mais refinados de processos que permitem a identificação de mecanismos subjacentes em ação. Esta tendência, verificada no âmbito internacional, começa, somente muito recentemente, a despertar o interesse dos pesquisadores brasileiros cuja produção, ainda incipiente na área, carece também, na maioria das vezes, de metodologia adequada a este tipo de investigação. Prevalecem ainda entre nós, as pesquisas de processo que se enquadram no modelo de "Estudo de Caso Tradicional" (Spence, 1993), que se baseiam em transcrições de sessões realizadas a partir da memória do terapeuta, que é também, na maioria das vezes, o responsável pela análise e interpretação dos resultados obtidos, e que, via de regra, se limita a corroborar premissas teóricas pré-existentes.

Este tipo de pesquisa, conforme o indica Spence (1993), encontra-se bastante desprestigiado na atualidade, na medida em que a visão do terapeuta é o único parâmetro possível para guiar os 
julgamentos e as conclusões. Além disso, o processo de coleta de dados se faz de uma forma assistemática e só se tem acesso àquilo que o terapeuta considerou relevante e decidiu incluir na apresentação do caso.

Como um desenvolvimento deste delineamento de pesquisa têm sido propostos novos procedimentos metodológicos que visam garantir maior objetividade no momento da coleta e do registro dos dados o que, por consequiência, amplia a confiança nos resultados e nas conclusões das pesquisas realizadas. Tratam-se de algumas diretrizes básicas para o encaminhamento do processo de observação, de descrição e de medida, capazes de permitir a identificação de fatores que tenham valor preditivo e que possam ser generalizados (Greenberg \& Newman, 1996).

No que respeita à observação defende-se a utilização de registros objetivos do processo terapêutico, tais como: o uso de áudio, ou mesmo de vídeo. Estes, possibilitam uma compreensão mais acurada do que efetivamente se passou na sessão, que pode ser exaustivamente estudada, seja pelo terapeuta seja por juízes independentes. Quando as avaliações são realizadas por juízes independentes são usadas, geralmente, categorias de análise operacionalmente definidas o que, por sua vez, permite a obtenção de bons índices da precisão dos julgamentos realizados.

De uma maneira geral, os registros em áudio e / ou vídeo são acompanhados de transcrições das sessões realizadas segundo padrões especificamente criados para a pesquisa em clínica, e que têm possibilitado diferentes leituras e interpretações de um mesmo material. Apenas para ilustrar, gostaríamos de citar a proposta do "Comitê sobre Atividades Científicas da Associação Psicanalítica Americana", cujo relatório final, apresentado por Klumpner \& Frank (1991), sugere normas e recomendações para a apresentação de material clínico. O objetivo é o de se procurar uma padronização na transcrição de sessões que respeite, o mais fielmente possível, o que se passou nela. Desta forma, como já sugerido, outras pessoas que não apenas o terapeuta, ou o paciente, podem acompanhar a evolução do processo.

A descrição exaustiva dos dados clínicos, procurando a reprodução mais fiel possível do que se passa no contexto das psicoterapias, favoreceu o desenvolvimento de instrumentos de medida baseados em análises intensivas do processo, e que têm permitido um melhor acompanhamento das interações terapeuta-paciente (Safran, Rice \& Greenberg, 1988).

Na medida em que mais de um pesquisador tem acesso a um determinado material, abre-se a possibilidade da existência de diferentes contribuições em tomo dele e, portanto, condições para um movimento convergente que viabiliza o acúmulo de conhecimento, tão necessário para o desenvolvimento da psicologia clínica.

Através do uso de escalas e instrumentos de medida baseados em análises intensivas de processos terapêuticos tem-se procurado:

1. explicar os mecanismos subjacentes através dos quais as mudanças ocorrem;

2. circunscrever os eventos bem definidos de mudança que tenham significância prática e teórica;

3. identificar num processo terapêutico específico, momentos de mudanças que possam ser generalizados (Greenberg, 1994).

As características acima sumarizadas, como já indicado, têm inspirado o desenvolvimento de um grande número de instrumentos de medida. Tratam-se, em geral, de instrumentos, que pela própria metodologia de aplicação e avaliação, devem ser utilizados por juízes independentes, adequadamente treinados no seu uso, garantindo-se com isto um grau de acordo estatisticamente 
significante.

Como exemplo deste tipo de instrumento pode-se citar, a "Rutgers Psychotherapy Progress Scale - RPPS" (Holland, Messer \& Roberts, 1996), que visa a avaliação do progresso de pacientes em psicoterapias psicodinâmicas, ou ainda, as "Defense Mechanisms Rating Scales" (peny,1990), desenvolvidas com a finalidade de fornecer medida precisa e válida para as manifestações clinicas dos mecanismos de defesa.

Uma outra característica deste tipo de instrumento é que eles são teoricamente orientados. Isto é, eles são desenvolvidos com o propósito de avaliar em que medida uma formulação teórica permite a compreensão de um determinado aspecto do processo terapêutico.

Uma terceira característica é a de que há uma valorização do contexto. Apesar de ainda persistirem as pesquisas que envolvem medidas que não levam em consideração o contexto, é crescente a preocupação em se propor medidas que visem ações dentro dele. Com isto pretende-se saber: que tipo de intervenção é mais útil em um contexto específico. Quando se considera o contexto, tem-se acesso ao sentido do que é comunicado e não apenas à forma. Conforme o defendem, Messer, Tishby e Spillman (1992), nestes casos, se o paciente diz, por exemplo, "está frio aqui dentro", fica mais

fácil identificar se isso corresponde a uma queixa velada, a um pedido, ou a uma crítica, tanto de um ponto de vista literal, quanto metafórico.

Por outro lado, nem todos os instrumentos de avaliação em pesquisa podem ou devem ser utilizados apenas para avaliações realizadas por juízes independentes. Na realidade, faz parte das orientações metodológicas atuais, relacionadas às pesquisas de mudança em processos terapêuticos, um procedimento chamado por Campbell e Fiske, de triangulação (1959, apud, Fonagy \& Moran, 1993 ).

A triangulação se refere ao procedimento de se incluir em uma mesma pesquisa medidas obtidas pelo terapeuta, por juízes independentes e pelo paciente. Provenientes de diferentes fontes, podem ser comparadas entre si, complementando-se umas à outras, confirmando-se, ou mesmo contrapondo-se entre si (Fonagy \& Moran, 1993). O objetivo, último, é o de garantir a possibilidade da formulação de um modelo explanatório mínimo que informe sobre a ocorrência de padrões recorrentes no processo terapêutico e que levem à comprensão dos fatores subjacentes à mudança (Luborsky, 1993 ).

Como exemplo de procedimento de avaliação do material clínico pelo terapeuta podemos citar, a "Escala Diagnóstica Adaptativa Operacionalizada - Redefinida" - EDAO-R (Simon, 1996a), proposta como uma ferramenta auxiliar para o clínico, na sua tarefa de avaliar e propor um determinado tipo de intervenção ou tratamento a alguém. Mas deve-se notar que, quando usada em pesquisa, além das avaliações do terapeuta, deve-se contar, igualmente, com avaliações realizadas por juízes independentes, garantindo-se, desta forma, a possibilidade de se avaliar o grau de precisão dos julgamentos

realizados. Embora inicialmente criada para orientar o psicodiagnóstico, a EDAO-R tem sido, igualmente, utilizada em diferentes momentos do processo terapêutico, como por exemplo: o meio do atendimento, o final ou o acompanhamento, fornecendo indicações sobre eventuais mudanças ocorridas na eficácia adaptativa do paciente (Yoshida e cols., 1994).

No que se refere à terceira fonte de avaliação que vem sendo utilizada em pesquisas de processo, aparecem, como já dissemos, as fornecidas pelos próprios pacientes através de 
instrumentos de avaliação auto-administrados (self-report). Nestes casos, pode-se investigar, por exemplo, a percepção do sujeito quanto: à remissão do sintoma, ao grau de satisfação obtido com o processo, à adequação da ajuda prestada pelo terapeuta em relação ao motivo da consulta, ou ainda, às fontes específicas de angústia que podem se constituir no foco do tratamento.

Com o propósito de ilustrar esta breve exposição sobre aspectos metodológicos em pesquisas de mudança em processos terapêuticos, apresentaremos, a seguir, mais detalhadamente, os instrumentos de avaliação citados acima, assim como alguns resultados de pesquisas em que eles foram utilizados.

\section{A "Escala Rutgers de Progresso em Psicoterapia"}

(Rutgers Psychotherapy Progress Scale . RPPS, 1992)

Esta escala, voltada para a avaliação de períodos de progresso em processos de psicoterapia, foi desenvolvida a partir do enfoque metodológico descrito acima Destina-se a pesquisas que envolvem o estudo intensivo de casos únicos, onde aspectos quantitativos e qualitativos da interação terapeuta paciente são avaliados contextualmente.

A RPPS consiste em um aprimoramento de uma versão anterior, intitulada "Rutgers Psychotherapy Progress and Stagnation Scale" - RPPSS (Messer,Tishby \& Spillman, 1992). Naquela versão, oito aspectos relacionados ao progresso do paciente e sete à sua estagnação foram inicialmente identificados na literatura psicanalítica e integravam a escala, que era avaliada por juízes independentes a partir da transcrição de sessões gravadas em áudio. O emprego desta escala em duas pesquisas (Messer Tishby \& Spillman,1992 e Tishby \& Messer, 1995) mostrou que, embora como um todo ela gozasse de boa fidedignidade, os itens relacionados à avaliação dos momentos de estagnação dentro do processo terapêutico não chegavam a ser avaliados. Uma outra limitação dizia respeito ao fato dela contar apenas com

um escore global final, resultado da indicação da presença ou ausência dos itens avaliados.

Na versão atual da escala (RPPS), os itens de estagnação foram suprimidos e cada item avaliado passou a contar com 5 pontos possíveis dentro de uma escala de tipo Likert. Além disto, um manual de avaliação, contendo os critérios de julgamento de cada item, foi desenvolvido (Rutgers Psychotherapy Progress Scale, 1992 ).

A RPPS conta com oito itens que visam medir respectivamente:

1. Expressão de material significante;

2. Desenvolvimento de insight;

3. Foco sobre a emoção;

4. Referência direta ao terapeuta e / ou à terapia;

5. Novo comportamento na sessão;

6. Colaboração;

7. Clareza e vivacidade na comunicação;

8. Foco no "Eu".

Cada item deve ser avaliado a cada bloco de cinco minutos da sessão, dando origem a um escore médio daquele item ao final da sessão e indicando o progresso verificado. Além disto, um escore total médio, envolvendo o somatório de todas as avaliações de cada item em todas as sessões, fornece uma indicação do progresso obtido pelo paciente na psicoterapia como um todo. Sendo assim, pode-se acompanhar quantitativa e qualitativamente o progresso do paciente ao longo 
da terapia e identificar momentos específicos da relação terapeutapaciente em que a ocorrência de variáveis específicas pode revelar características importantes relacionadas com a mudança.

Para estimar o grau de precisão, de validade preditiva e a estrutura fatorial da RPPS, partiu-se de dois processos de psicoterapias breves psicodinâmicas avaliados pelos respectivos pacientes, terapeutas e por clínicos independentes. Um processo foi considerado como bem sucedido e o outro como tendo resultados medianamente positivos (Roberts, Messer \& Holland, 1996). Tratavam-se de pacientes do sexo feminino com queixa de ansiedade e dificuldades de relacionamento. Com base nas avaliações realizadas por quatro juízes independentes, a RPPS demonstrou possuir um bom índice de acordo entre avaliadores, além de consistência interna. Quanto à validade preditiva, verificou-se que em seis dos oito itens, assim como no escore total, os escores eram significantemente superiores no caso considerado bem sucedido, quando comparado ao medianamente bem sucedido. Em relação à análise da estrutura fatorial, a rotação varimax realizada com os escores de cada processo terapêutico indicou a presença de três fatores com semelhanças e diferenças entre os dois processos.

Os resultados de investigação da validade de constructo da RPPS foram apresentados em outro artigo destinado para este fim (Holland, Messer e Roberts, 1996). Nele, os autores, utilizando os dois casos clínicos que já haviam dado origem ao trabalho descrito acima, examinaram as correlações da RPPS com uma outra escala voltada para a avaliação de processo em psicoterapia, a "Vanderbilt Psychtotherapy Process Scale - VPPS"(O'Malley, Suh \& Strupp, 1983). Verificaram inicialmentea as correlações entre a RPPS e a VPPS de cada caso para ver se as relações entre elas eram as mesmas nos dois processos. Justificam tal procedimento referindo-se à recomendação feita por Hilliard (1993), segundo a qual, em pesquisas envolvendo casos únicos, deve-se examinar primeiro os dados de cada caso antes de agregá-los. Em geral, os resultados revelaram validade convergente e discriminante da RPPS, além de validade concorrente de itens individuais. Como conclusão, os autores admitem a existência de evidências da precisão e da validade da RPPS.

No presente, encontram-se em andamento duas outras pesquisas envolvendo a RPPS. A primeira delas deverá constituir-se na tese de doutorado de Enéas (1996), e pretende verificar a precisão e a validade preditiva da RPPS em processos de psicoterapia breve. A segunda, realizada por Yoshida (1998), deverá avaliar diferentes aspectos do processo de mudança em psicoterapias breves psicodinâmicas, incluindo avaliações dos mecanismos de defesa e da configuração adaptativa, através das escalas apresentadas a seguir: "Defense Rating Mechanism Scales"- DRMS (Perry, 1990) e "Escala Diagnóstica Adaptativa Operacionalizada Redefinida" - EDAO-R (Simon, 1996a).

\section{As "Escalas Clínicas de Avaliação dos Mecanismos de Defesa"}

(Defense Mechanism Rating Scales . DMRS) ${ }^{2}$ (Perry, 1990)

No âmbito da pesquisa de psicoterapias psicodinâmicas, a possibilidade de mensuração e

\footnotetext{
${ }^{2}$ Deve-se esclarecer, que as "Escalas Clínicas de Avaliação dos Mecanismos de Defesa" foram inicialmente concebidas para a avaliação qualitativa dos mecanismos de defesa predominantes do paciente. Diante do material clínico a ser avaliado. geralmente a transcrição de uma sessão de terapia ou entrevista diagnóstica, acompanhada de vídeo, os juízes deveriam opinar pela ausência definitiva, uso provável ou uso definitivo de cada um dos 28 mecanismos de defesa descritos. E dentro desta concepção. cada um era visto como uma escala. Esta é a razão para o nome do instrumento aparecer no plural na sua versão em inglês (Perry, 1990), o que foi mantido na tradução para o português (Wiethaeuper e Yoshida, 1996). Contudo, no presente trabalho, referimo-nos a ele usando a sigla DMRS, no singular, para facilidade de discurso.
} 
avaliação dos mecanismos de defesa é especialmente relevante se se considera o papel que eles desempenham no que foi chamado de "triângulo de conflito" (Malan, 1979 11983), e que, juntamente com a "tríade de pessoa", constitui o ponto de partida das intervenções do terapeuta.Conforme o refere Malan (1979 / 1983, p. 114), "o alvo de quase toda a psicoterapia dinâmica consiste em chegar, por debaixo da defesa e da ansiedade, até o sentimento encoberto, e então investigar este sentimento recuando do presente até suas origens no passado, geralmente na relação como os pais" (grifo do autor).

O conceito de defesa proposto por Freud (1894 / 1973), já nos seus primeiros escritos sobre as psiconeuroses (por ex.: dissociação e repressão), mantém-se até o presente e continua a inspirar a pesquisa. Na concepção de Freud, os mecanismos de defesa teriam uma função de proteção ao indivíduo, ameaçado em seu equilíbrio psíquico por emoções, idéias e impulsos, que lhe trariam sofrimento.

Autores mais recentes, têm destacado o papel adaptativo das defesas que modulariam o comportamento do sujeito em função das demandas do meio, das exigências superegóicas e das necessidades impulsivas (Vaillant, 1992). Como resultado da ação dos diferentes mecanismos de defesa, o comportamento manifesto assumiria, portanto, configurações passíveis de serem classificadas como: mais ou menos patológicas, mais ou menos maduras, mais ou menos adaptativas. E, dentro desta perspectiva, à medida que ocorre a mudança no processo terapêutico, espera-se, ao menos teoricamente, que a configuração das defesas utilizadas pelo paciente também se modifique.

Como uma maneira de tomar acessível à pesquisa empírica estas concepções teóricas, de uso tão difundido na clínica psicológica, observam-se tentativas de operacionalizá-las. Há propostas de instrumentos de avaliação dos mecanismos de defesa que propõem sua hierarquização segundo a qualidade da adaptação geral do sujeito (Peny, 1993). Como exemplo de instrumento autoadministrado pode-se citar, o "Bond's Defense Style Questionnaire (DSQ)", presente no livro de Vaillant (1992). E, como exemplo de instrumento que depende de avaliadores externos, há as "Escalas Clínicas de Avaliação dos Mecanismos de Defesa (DMRS)", que serão apresentadas mais extensamente neste texto.

No caso específico dos mecanismos de defesa, existe uma tendência a se preferir os instrumentos de avaliação. dependentes de juízes externos pois se argumenta que os auto-administrados estariam limitados pela capacidade do sujeito de descrever e identificar seus respectivos padrões defensivos típicos, que por definição, são inconscientes e, portanto, inacessíveis, naquele momento, para o sujeito (Jacobson e cols, 1986).

Como a RPPS, a DMRS foi desenvolvida com objetivo de pesquisa envolvendo material clínico. Na sua versão original, proposta por Perry e Cooper (1986), foi aplicada a amostra composta de sujeitos diagnosticados como apresentando distúrbio de personalidade borderline e distúrbio de personalidade antisocial. Na ocasião, a escala era composta de 22 defesas que foram utilizadas por juízes leigos a partir da observação de entrevistas psicodinâmicas realizadas com os sujeitos da amostra. A precisão das defesas individuais foi considerada aceitável quando se comparou as avaliações consensuais dos grupos de avaliadores (precisão intraclasse média $=.57$ ). Ela foi mais alta (valor médio $=.74$ ), quando se agrupou as defesas pela função que elas desempenham na dinâmica do indivíduo: ação, borderline, evitação (disawoa/), narcisistas e obsessivas. 
Na sua atual versão (5a ed.), a DMRS consta da definição de 28 mecanismos de defesa, inclui a função de cada um, comentários sobre como discriminá-los de outras defesas e uma escala de três pontos onde:

1. corresponde à ausência de ocorrência do mecanismo;

2. à sua presença provável e

3. à sua presença definitiva. Está apresentada em um manual (Perry,1990), que prescreve uma forma de avaliação qualitativa e outra quantitativa dos mecanismos de defesa utilizados por pacientes em entrevistas psicológicas e sessões de psicoterapias gravadas em áudio e / ou vídeo e transcritas. Uma tradução para o português já se encontra disponível (Wiethaeuper \& Yoshida, 1996).

De acordo com o procedimento prescrito para a avaliação qualitativa, juízes treinados devem identificar, ao final da entrevista, os mecanismos predominantemente utilizados pelo paciente, após opinarem pela ausência, presença provável ou presença definitiva de cada um dos mecanismos definidos na escala. No caso da avaliação quantitativa, devem indicar os trechos das transcrições que evidenciam as manifestações dos mecanismos de defesa, nomeando-os.

Na escala, "as defesas estão divididas em 7 grupos hierarquicamente arranjados, sendo que a cada um é dado um número que representa o peso dado a estas defesas. Dentro de cada grupo, somam-se os escores de cada defesa, resultando num subtotal para aquele grupo... Cada subtotal é então multiplicado pelo peso respectivo. Os sete subtotais são somados (a), e os sete pesos das defesas também (b). O Escore Global de Maturidade Defensiva (c) é a, média dos pesos, ou b dividido por a. Este número varia de 1.00 a 7.00." (Perry,1990, p. 4).

Estão previstos os seguintes níveis de funcionamento das defesas: maduras, obsessivas, neuróticas, narcisistas, evitação, borderline e de ação. Correspondem às defesas maduras: a afiliação, o altruísmo, a antecipação, o humor, a auto-afirmação, a auto-observação, a sublimação e a supressão. São consideradas defesas obsessivas: o isolamento, a intelectualização e a anulação. Como defesas neuróticas há: a repressão, a dissociação, a formação reativa e o deslocamento. Como defesas narcisistas: a onipotência, a idealização e a desvalorização. Dentre as de evitação: a negação, a projeção, a racionalização e a fantasia. Como defesas borderline aparecem: a cisão (das imagens do outro), a cisão (das imagens de si) e a identificação projetiva. E, como defesas de ação: o agir (acting out), a agressão passiva e a hipocondria.

Com base na proposta de hierarquização das defesas, pesquisas foram feitas procurando comparar o nível das defesas utilizadas por sujeitos com diagnósticos diferentes de acordo com o DSM-III-R (American Psychiatric Association, 1987). Por exemplo, Block e cols. (1993) apresentam um estudo piloto em que a DMRS foi usada para a avaliação psicodinâmica de pacientes distímicos e com desordem do pânico. Os resultados mostram que embora os dois grupos de pacientes apresentem primordialmente defesas dos níveis mais baixos de maturidade, os distímicos apresentam escores significantemente mais elevados de defesas narcísicas, de evitação e de ação.

Em outra pesquisa, Perry e Cooper (1989) verificaram a asssociação entre medidas da DMRS e do funcionamento global medido através da "Global Assessment Scale" - GAS. A amostra, composta de pacientes com distúrbio de personalidade borderline, pacientes com distúrbio antissocial e com distúrbio afetivo de tipo bipolar-II, foi avaliada em dois momentos distintos: no momento em que o sujeito buscou os serviços clínicos do Hospital Cambridge, a partir de anúncio 
colocado em jornal, e depois, em várias avaliações realizadas ao longo de acompanhamento realizado por vários anos, em que a estabilidade do quadro psicopatológico era verificada. Os resultados mostraram que as defesas imaturas estavam associadas com índices mais elevados de sintomas, funcionamento global mais pobre e maior proporção de tempo em que o desempenho de papéis psicossociais estava prejudicado.

Em nosso meio, encontra-se em fase final de coleta de dados, pesquisa realizada por Gatti (1998) que visa obter índices de precisão e validade concorrente da DMRS. Para o estudo de validade concorrente, avaliações da DMRS de entrevistas realizadas com estudantes universitários, serão correlacionadas a medidas da eficácia adaptativa, obtidas através da EDAO-R e a medidas de saúde mental, obtidas através da adaptação brasileira do QSG (Questionário de Saúde Geral de Goldberg) (Goldberg, 1972 /1996).

Quanto ao emprego da DMRS voltado para a identificação de mudanças em processos terapêuticos, encontra-se em curso na U. de Montréal, pesquisa de St-Amand (1995), em que o autor pretende identificar configurações ou padrões recorrentes de mecanismos de defesa utilizados por uma paciente em psicoterapia breve. No mesmo espírito há pesquisa, já mencionada acima de Yoshida (1998), ainda em fase inicial de coleta de dados, que deverá envolver outras medidas de mudança.

\section{A "Escala Diagnóstica Adaptativa Operacionalizada-Redefinida" EDAO-R (Sirnon. 1996a)}

Assim como alterações na configuração dos mecanismos de defesa podem nos informar sobre o rumo das mudanças vividas pelo sujeito ao longo de um processo terapêutico, alterações na qualidade das respostas adaptativas constituem um indício de mudanças de seu funcionamento geral. A idéia é a de que mudanças na eficácia adaptativa correspondam a mudanças na qualidade da saúde mental, e vice-versa. Desta forma, instrumentos de medida capazes de informar sobre a eficácia com que o sujeito está respondendo às contingências a que está exposto, podem ser de grande utilidade em pesquisas voltadas para a identificação de mudanças em processos terapêuticos.

Em nosso meio, dispomos da EDAO-R (Simon, 1996a), que constitui a segunda versão da original, EDAO (Simon,1983). Desde que foi proposta, a EDAO vinha sendo aplicada aos mais diversos tipos de população dentro dos mais diferentes contextos (por ex.: Calejon, 1996; Rosa,1996; Rosa,!., 1996; Gandini,1995; Coellho Filho, 1995; Yoshidae cols., 1994). Na prática da pesquisa, contudo, começou e evidenciar algumas contradições quanto à capacidade de discriminar pessoas com configurações adaptativas diferentes, levando seu idealizador a propor uma redefinição do sistema de pontuação de suas categorias (Simon, 1996a).

Na primeira versão da escala, a eficácia adaptativa era medida a partir da avaliação da adequação das respostas do sujeito ao setor Afetivo-Relacional (AR), da Produtividade (Pr), SócioCultural (SC) e Orgânico (Or). Para cada setor, o avaliador considerava a adequação das respostas segundo três critérios:

1. o grau de satisfação propiciado;

2. a medida em que constituia solução para a situação enfrentada; e

3. o grau em que se compatibilizava ou não com as normas culturais.

Quando em um setor predominassem respostas que atendessem aos três critérios, a qualidade da adaptação daquele setor seria adequada; se apenas dois destes critérios fossem satisfeitos seria 
pouco-adequada; e se apenas um dos critérios fosse satisfeito, pouquissimo adequada. Seguindo estes critérios, foi desenvolvida uma listagem com as repostas mais freqüentemente encontradas em população de calouros da Escola Paulista de Medicina, organizada por setores, e que constituía a Escala propriamente dita (Simon, 1983).

Desenvolvida portanto, empiricamente, a EDAO destinava-se à avaliação de populações de" jovens e adultos (não idosos); b) pertencentes à classe média inferior para cima; c) habitantes da zona urbana brasileira; d) participantes dos valores e costumes da civilização ocidental (Simon, 1983, p. 88). O seu emprego se dava a partir do confronto dos dados obtidos em entrevista psicológica individual com as respostas previstas na Escala. Como esta última não pretendia ser exaustiva, este confronto pautava-se principalmente em julgamento clínico. Uma vez avaliados os setores, passava-se para a classificação geral da adaptação, com a especificação do grupo a que pertencia o sujeito.

Estavam previstos seis grupos para a classificação da eficácia da adaptação, sendo: três para a adaptação estável e três para a adaptação na crise. Tinha-se respectivamente: Gr.I adaptação eficaz; Gr.III - adaptação não-eficaz moderada; Gr.V - adaptação não-eficaz severa; Gr.II adaptação eficaz em crise.; Gr.IV adaptação não-eficaz moderada em crise; Gr.VI adaptação não-eficaz severa em crise. O Gr.VII destinava-se a casos em que a avaliação era impossível. O processo de avaliação da adaptação terminava com o registro dos dados relevantes por setor; a qualidade da adaptação também por setor e a indicação do grupo, que correspondia à configuração adaptativa geral.

Conforme, já referido, desde sua concepção, a EDAO passou a ser usada em diferentes contextos e populações. Como conseqüência, a listagem original de respostas, que constituía a Escala propriamente dita, não se aplicava a muitos casos em que as características da amostra estudada eram diferentes daquelas apresentadas pelos estudantes universitários que compuseram a amostra original. Nestas situações, passou-se a avaliar os setores com base apenas nos três critérios de avaliação da qualidade das respostas dos sujeitos, sem depender de listagens de respostas. Esta modificação de concepção da EDAO, conferiu-lhe maior versatilidade uma vez que, desta forma, ela podia ser aplicada a qualquer tipo de população.

Embora mais versátil, ela carecia de capacidade de discriminação adequada dos sujeitos com adaptação não eficaz. Daí a razão da proposta de sua redefinição. Nesta versão, prevaleceu idéia defendida por Simon (1996b), segundo a qual, o setor AR ocupa posição central no conjunto da adaptação e, portanto, tem um peso diferente dos demais na determinação da eficácia adaptativa. $\mathrm{N}$ esta medida, a redefinição da Escala passa pelo redimensionamento qualitativo da avaliação da adaptação, procurando fazer juz à observação empírica e facilitar a orientação do clínico na escolha do procedimento psicoterápico mais adequado ao paciente. Esta observação, acompanhada do reconhecimento da importância da Produtividade na configuração adaptativa dos sujeitos, facultou a Simon, a concepção da EDAO-R.

De acordo com esta nova versão, só são quantificados os setores Afetivo - Relacional (AR) e o da Produtividade (Pr). Para o primeiro, são propostas as seguintes pontuações: 3, para adequado; 2 para pouco adequado e 1 para pouquíssimo adequado. Quanto ao segundo, as pontuações seriam, respectivamente: 2 , 1 e 0.5 .

Da combinação das pontuações possíveis decorrem os cinco grupos seguintes:

Grupo 1. Adaptação eficaz, quando AR e Pr são adequados;

Grupo 2. Adaptação ineficaz leve, quando um dos setores é adequado e o outro é pouco 
adequado;

Grupo 3. Adaptação ineficaz moderada, quando ambos são pouco adequados, ou um é adequado e o outro pouquíssimo adequado;

Grupo 4. Adaptação ineficaz severa, quando um setor é pouco e o outro pouquíssimo adequado;

Grupo 5. Adaptação ineficaz grave, quando ambos são pouquíssimo adequados.

Para esta nova versão, Simon (1996, p.11) sugere ainda uma "descrição fenomenológica para facilitar o entendimento clínico da Escala". Segundo ela, a adaptação eficaz (Gr. 1) corresponde à personalidade dita "normal, em que há"raros sintomas neuróticos ou caracterológicos". À adaptação ineficaz leve (Gr.2) corresponderiam "sintomas neuróticos brandos, ligeiros traços caracterológicos, algumas inibições". À adaptação ineficaz moderada (Gr.3), "alguns sintomas neuróticos, inibição moderada, alguns traços caracterológicos". À adaptação ineficaz severa (Gr. 4), "sintomas neuróticos mais limitadores, inibições restritivas, rigidez de traços caracterológicos". E finalmente, à adaptação ineficaz grave (Gr. 5), corresponderiam "neuroses incapacitantes, borderline, psicóticos não agudos, extrema rigidez caracterológica".

Quanto aos setores SC e 01' estes têm um papel mais qualitativo na EDAO - R, auxiliando no diagnóstico discriminativo individual ou na orientação psicoterápica (Simon, 1996a).

Em relação às qualidades psicométricas desta versão, pesquisa voltada para o estabelecimento da precisão e da validade da EDAO-R (Yoshida, 1997) indicou um alto grau de acordo entre juízes independentes. Constatou ainda, que ela pode ser utilizada como medida auxiliar para discriminar sujeitos com maior probabilidade para abandonar processos psicoterapêuticos breves dos com maior probabilidade para concluí-los.

Em relação à possibilidade de acompanhamento de mudanças na configuração adaptativa dos sujeitos durante processos terapêuticos, existe, no momento, apenas uma expectativa, posto que pesquisa com este fim, ainda se encontra em andamento (Yoshida, 1998).

\section{Considerações finais}

Finalizando este trabalho introdutório sobre avaliação de mudanças em processos terapêuticos, gostaríamos de enfatizar a necessidade de se contar com procedimentos rigorosos em todas as fases do processo, além de se procurar utilizar avaliações realizadas a partir de diferentes perspectivas (terapeuta,juízes e paciente), tendo-se com isto a garantia de uma visão mais abrangente e integrada dos múltiplos fatores envolvidos em processo tão complexo como é o de qualquer psicoterapia.

Por outro lado, devemos nos ocupar com a convergência dos esforços de diferentes pesquisadores, em torno de procedimentos metodológicos e de instrumentos de avaliação comuns, criando-se com isto, uma base comum para a construção de um conhecimento que seja empiricamente formulado.

\section{Referências Bibliográficas}

American Psychiatric Association (1987). Diagnostic and statistical manual of mental disorders (3a. ed. rev.). Washington, DC: American Psychiatric Association. 
Block, A. L., Shear, M. K., Markowitz, 1. C., Leon, A. c., Perry, J. P. (1993). An empirical study of defense mechanism in dysthimia. American Journal of Psychiatry, 150 (8), 1194-1198.

Calejon, L. M. (1996). Estudos com pacientes de clínica psicológica universitária. Mudanças: psicoterapia e estudos psicossociais,3 (3/4), 235-258.

Coelho Filho, 1. G. (1995). Processo e aliança terapêutica de pacientes borderline em psicoterapia breve psicodinâmica. Dissertação de Mestrado, PUCCAMP, Campinas.

Enéas, M. L. E. (1996). Escala Rutgers de Progresso em Psicoterapia: precisão e validade preditiva. Projeto de Tese de Doutorado, PUCCAMP, Campinas.

Fonagy, P. \& Moran, G. (1993). Selecting single case research designs for clinicians . In N. E. Miller, L., Luborsky, J. P., Barber \& J. P., Docherty (Eds), Psychodynamic treatment reearch: $a$ handbookfor clinical practice (pp. 6295). New Y ork : Basic Books.

Freud, S. (1973). Las neuropsicosis de defensa. In, S.Freud, Obras Completas (pp. 169-177). (L-R de Torres Trad.). Madrid: Editorial Biblioteca Nueva (Originalmente publicado em 1894).

Gandini, R. C. G. (1995). Câncer de mama: evolução da eficácia adaptativa em mulheres mastectomizadas. Tese de Doutorado, IPUSP, São Paulo.

Gatti, A. L. (1996). Escalas de Avaliação dos mecanismos de defesa: precisão e validade concorrente. Projeto de Tese de Doutorado, PUCCAMP, Campinas.

Goldberg, D. P. (1996). Questionário de Saúde Geral de Goldberg: manual técnico QSG (adaptação brasileira). (L. Pasquali, V. V. Gouveia, W. R Andriola, F. J. Miranda, A. L. M. Ramos, Trads.) São Paulo: Casa do Psicólogo.

Goldfried, M. R. \& Wolfe, B. E. (1996). Psychotherapy practice and research: repairing a strained alliance. American Psychologist, 51 ( 10), 1007-1016.

Greenberg, L. S. (1994). The investigation of change its measurement and explanation. In R. L. Russell (Ed.), Reassessing psychotherapy research (pp. 114-143). New York: The Guilford Press.

Greenberg, L. S. \& Newman, F. L. (1996). An approach to psychotherapy change process research: introduction to the special section. Journal of consulting and clinical psychology, 64 ( 3), 435 438.

Hilliard, R. B. (1993). Introduction to special section: single-case research in psychotherapy. Journal of Consulting and Clinical Psychology,61 (3), 371-372.

Holland, S. J., Messer, S. R \& Roberts, N. E. (1996). Construct Validity of the Rutgers Psychotherapy Progress Scale. in mimeo.

Horowitz, L. M., Rosenberg, S. E., Bauer, R A., Ureno, G. \& Villasenor, V. S. (1988). Inventory of Interpersonal Problems: psychometrics properties and clinical applications. Journal of Consulting and Clinical Psychology, 56, 885892.

Jacobson, A. M., Beardslee, W., Hauser, S. Noam, G. G., Powers, S. I. , Houlihan, J. \& Rider, E. (1986). Evaluating ego defense mechanisms using clinical interviews: an empirical study of 
adolescent diabetic and psychiatric patients. Journal of Adolescence,9. 303-319.

Klumpner, G. H. \& Frank, A. (1991). On methods of reporting clinical material. Journal of the American Psychoanalytic Association, 39, 537- 551.

Lambert, M. J. (1991). Introduction to psychotherapy research. In L. E. Beutler \& M. Crago (Eds.), Psychotherapy research: an international review of programmatic studies. (pp. 1-12). Washington, DC: APA.

Luborsky, L. (1993). Documenting symptom formation during psychotherapy. In N. E. Miller, L. Luborsky, 1. P. Barber \& 1. P. Docherty (Eds), Psychodynamic treatment research: a handbook for clinical practice,(pp. 3 - /3). N ew Y ork : Basic Books.

MaIlan, D. H. (1983). Psicoterapia individual a ciência da psicodinâmica. (Maria Clarissa Juchem Trad.). Porto Alegre: Artes Médicas. (Originalmente publicado em 1979).

Messer, S. B. \& Wan"en, C. S. (1995). Models of brief psychodynamic therapy: a comparative approach. New Y ork: Guilford Press.

Messer, S. B., Tishby, O. \& Spillman, A. (1992). Taking context seriously in psychotherapy research: relating therapist interventions to patient progress in brief psychodynamic therapy. Journal ofConsulting and Clinical Psychology. 60 (5), 678-688.

O'Malley, S. S., Suh, C. S. \& Strupp, H. H. (1983). The Vanderbilt Psychotherapy Process Scale: a report of the scale development and a process outcome study. Journal of Consulting and Clinical Psychology.51 (4), 581-586.

PefI)', J. C. (1993). Defenses and their effects. In N.E. Miller, L. Luborsky, J.P. Barber \& 1. P. Docherty (Eds.). Psychodynamic treatment research: a handbook for clinical practice.(pp. 274306). New York: Basic Books.

PefI)', 1. C. (1990). Defense Mechanism Rating Scales. 5th ed., in mimeo.

Perry,1. C. \& Cooper, S. H. (1986). A preliminary report on defense and conflits associated with borderline personality disorder. Journal of the American Psychoanalitic Association. 34. 863893.

Roberts, N. E., Messer, S. B. \& Holland, S. (1996). The reliability. validity, and factor structure of the Rutgers Psychotherapy Progress Scale, in mimeo.

Rogers, C. R. (1974). Terapia centrada no paciente. (M. C. Ferreira Trad.) Lisboa: Moraes Editores. (Originalmente publicado em 1951).

Rosa, L P. (1996). Estudo do grau de adaptação em um grupo de pacientes anorgásmicas. Mudanças: psicoterapia e estudos psicossociais,3 (3/4), 111-130.

Rosa, 1. T. (1996). Contribuições da EDAO à psicologia clínica preventiva: ensino, clínica e pesquisa. Mudanças: psicoterapia e estudos psicossociais,3 (3/4), 109-110.

Rutgers Psychotherapy Progress Scale - RPPS (1992). Scoring Manual, in mimeo.

Safram, J. D., Rice, L. N. \& Greenberg, L.S. (1988). Integrating psychotherapy research and 
practice: modeling the change processo Psychotherapy, 25,1-17.

Simon, R. (1996a). Proposta de redifinição da E. D. A. O. Escala Diagnóstica Adaptativa Operacionalizada, in mimeo.

Simon, R. (1996b). Do diagnóstico à psicoterapia breve. Jornal Brasileiro de Psiquiatria,445 (7), 403-408.

Simon, R. (1983). Psicologia clínica preventiva: novos fundamentos. São Paulo: Vetor.

Spence, D. P. (1993). Traditional case studies and prescriptions for improving them. In N. E. Miller, L. Luborsky, J. P. Barber \& J. P. Docherty (Eds), Psychodynamic treatment research: a handbook for clinical practice, (pp. 37 - 52). New York: Basic Books.

St-Amand, P. (1995). Une analyse du processus de l'activité défensive du moi du patient en psychothérapie. Projeto de Tese de Doutorado, U. Montréal, Montréal.

Tishby, O. \& Messer, S. B. (1995). The relationship between plan compatibility of therapist interventions and patient progress: a comparison of two plan formulations. Psychotherapy Research, 5 (1), 76-88.

Vaillant, G. E. (1992). Ego mechanisms of defense: a guide for clinicians and researchers. Washington: American Psychiatric Press.

Wiethaeuper, D. \& Yoshida, E. M. P. (1996). Escalas de avaliação dos mecanismos de defesa. 5a. edição, in mimeo.

Y oshida, E. M. P. (1998). Avaliação de mudança em psicoterapia breve psicodinâmica. Projeto de Pesquisa, PUCCAMP, Campinas.

Yoshida, E. M. P. (1997). EDAO-R: precisão e validade. Submetido ao Boletim de Psicologia, in mimeo.

Yoshida, E. M. P., Enéas, M. L. E., Vasconcelos, A. S., Rillo, C. \& Duarte, K. M. (1994). Psicoterapia psicodinâmica breve: estudo de acompanhamento. Boletim de Psicologia, 44 (100 / 10 1),6168. 